\title{
The Use of Stereomicroscopy in Conjunction with In Situ Straining TEM for Studying Dislocation Behavior
}

\author{
Rodney J. McCabe*, Amit Misra*, Terence E. Mitchell* \\ *MS-G755, Los Alamos National Laboratory, Los Alamos, NM 87545
}

The ability to monitor dynamic motion and interactions of dislocations in three dimensions in the TEM would be beneficial for enhancing the understanding of dislocation behavior. Such capabilities would also be useful for direct input and validation of $3 \mathrm{D}$ discrete dislocation dynamics models. The present study employs the use of stereomicroscopy in combination with in situ straining TEM to study dislocation behavior. The experiments involve obtaining a 3D description of dislocation configurations including Burgers vectors using a modified stereo-TEM technique that is usable with a single tilt (in situ) TEM holder. Following the 3D analysis, the specimen is strained in situ in the microscope and the dislocation motion and interactions are recorded. Following deformation, a 3D description of the final dislocation microstructure is obtained. Although direct 3D observations of dislocation motion is not possible, knowledge of the $3 \mathrm{D}$ configuration preceding and following deformation along with the $2 \mathrm{D}$ record of the motion allows for a reasonable description of the $3 \mathrm{D}$ behavior.

Conventional stereomicroscopy of crystalline materials is almost never possible with a single tilt TEM holder making it unfeasible for most commercial in situ TEM holders. A modified stereo technique was developed in order to utilize stereo-TEM using single tilt holders. The technique is a weak beam technique demonstrated in Figure 1 involving changing the sign of $\mathbf{g}$ and/or $\mathrm{s}_{\mathrm{g}}$ while tilting across a Kikuchi band that is at less than around $30^{\circ}$ to the tilting direction. For dislocations, a change in either $\mathbf{g}$ or $\mathrm{s}_{\mathrm{g}}$ results in a shift in the peak in image intensity with respect to the dislocation core position [1]. To avoid errors associated with this shift, the ideal stereo conditions for this technique are for both $\mathbf{g}$ and $\mathrm{s}_{\mathrm{g}}$ to change signs between the two stereo images.

An example of the use of stereo-TEM in conjunction with in situ straining microscopy for an annealed copper sample is given in Figures 2 and 3. Figure 2 is a sequence showing dislocation motion recorded during in situ straining. Figure 3 is the 3D analysis of the before and after configurations of three of the dislocations. The before data comes directly from the micrographs in Figure 1. The stereo analysis was performed using Sterecon, a 3D reconstruction software developed at Wadsworth Laboratories, Albany, NY [2]. Using the 3D analysis, the in situ record, and knowledge of the active slip systems for the dislocations, it is possible to deduce that the dislocations numbered 1 and 2 do not intersect during the in situ portion of the experiment even though they appear to in the $2 \mathrm{D}$ projection [3].

\section{References}

[1] Cockayne DJH. J. Microsc. 98 (1973) 116-134

[2] Marko , M. and A. Leith. Sterecon -- Three-Dimensional Reconstruction from Stereoscopic Contouring. J. Structural Biol, 116: (1996) 93-98.

[2] This research is funded by the US Department of Energy, Office of Basic Energy Sciences. 

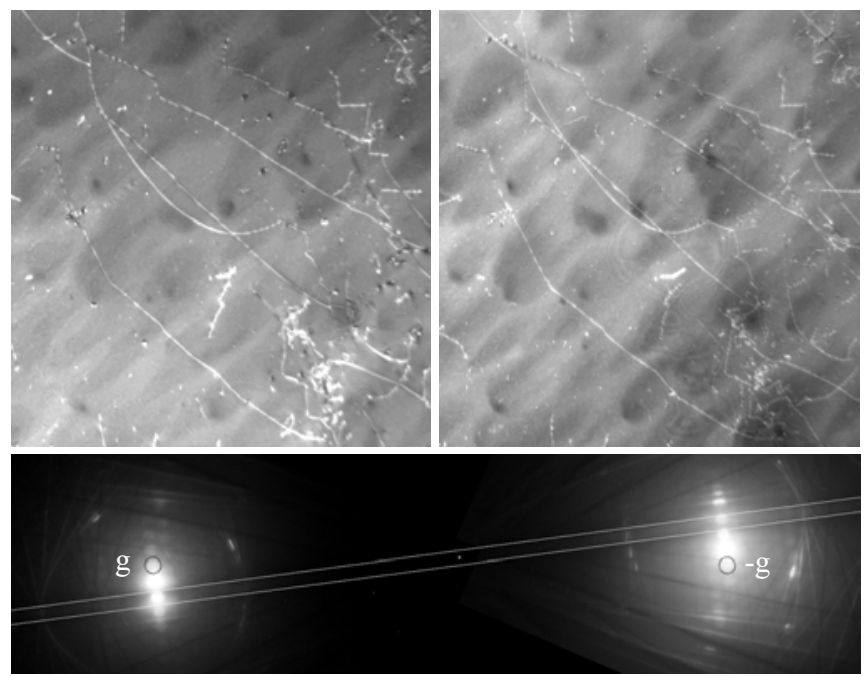

Figure 1. Stereo-pair obtained with the modified stereo technique. In the left image, $\mathbf{g}$ is used for imaging and $-2 \mathbf{g}$ is near the Bragg condition. In the right image, $\mathbf{- g}$ is used for imaging and $\mathbf{3} \mathbf{g}$ is near the Bragg condition.

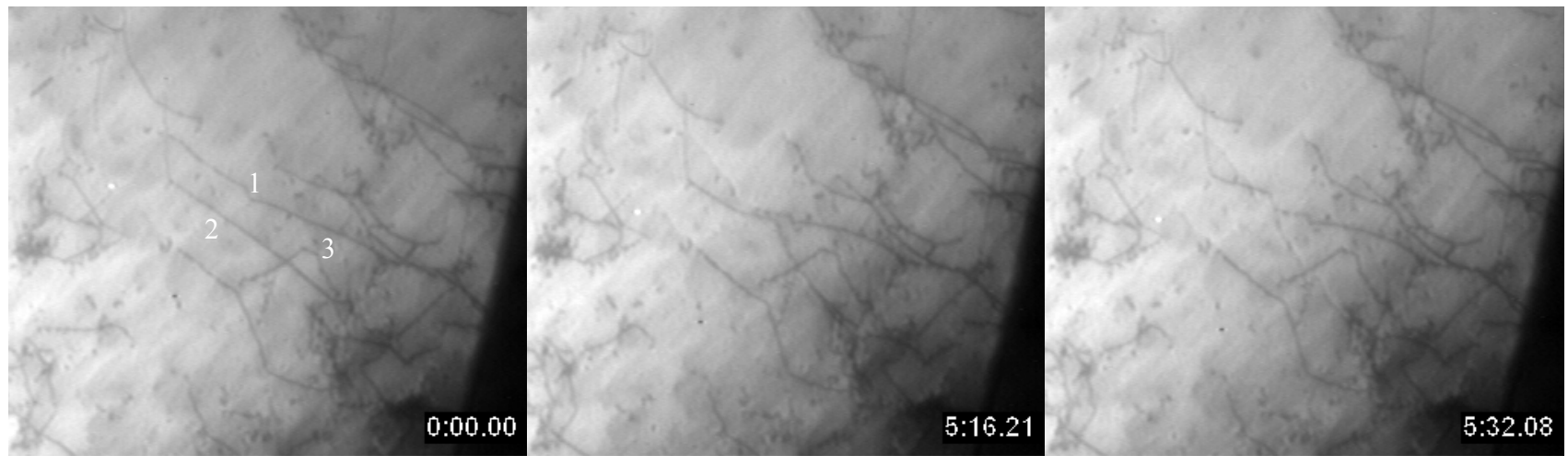

Figure 2. Progression of images during in situ loading. From these $2 \mathrm{D}$ projections, dislocations 1 and 2 appear to possibly intersect.

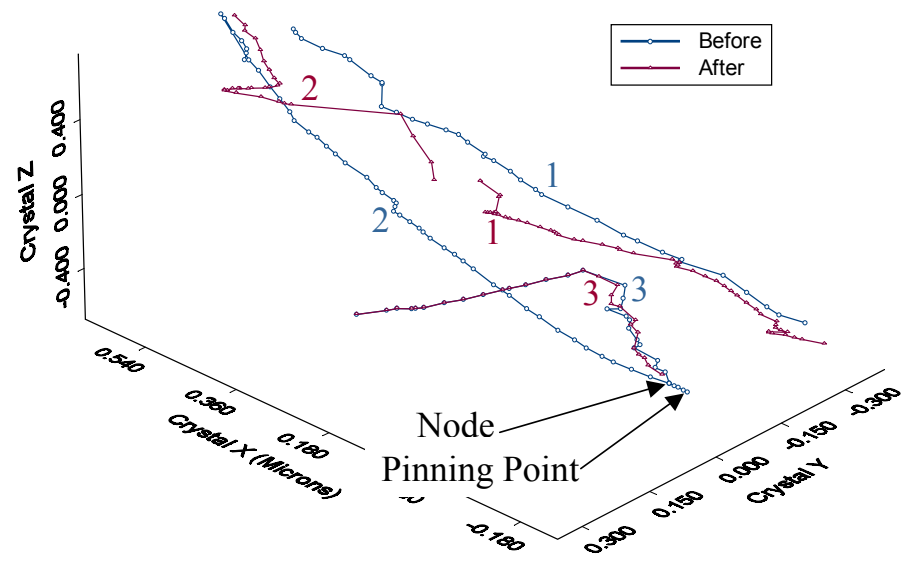

Figure 3. Before and after $3 \mathrm{D}$ representation of some notable dislocations. 